\title{
MINIMIZING THE DIRICHLET ENERGY OVER A SPACE OF MEASURE PRESERVING MAPS
}

\author{
Ali TAHeRI
}

Abstract. Let $\Omega \subset \mathbb{R}^{n}$ be a bounded Lipschitz domain and consider the Dirichlet energy functional

$$
\mathbb{F}[u, \Omega]:=\frac{1}{2} \int_{\Omega}|\nabla u(x)|^{2} d x,
$$

over the space of measure preserving maps

$$
\mathcal{A}(\Omega)=\left\{u \in W^{1,2}\left(\Omega, \mathbb{R}^{n}\right):\left.u\right|_{\partial \Omega}=x, \operatorname{det} \nabla u=1 \mathcal{L}^{n} \text {-a.e. in } \Omega\right\} .
$$

Motivated by their significance in topology and the study of mapping class groups, in this paper we consider a class of maps, referred to as twists, and examine them in connection with the Euler-Lagrange equations associated with $\mathbb{F}$ over $\mathcal{A}(\Omega)$. We investigate various qualitative properties of the resulting solutions in view of a remarkably simple, yet seemingly unknown explicit formula, when $n=2$.

\section{Introduction}

Let $\Omega \subset \mathbb{R}^{n}$ be a bounded Lipschitz domain and consider the Dirichlet energy functional

$$
\mathbb{F}[u, \Omega]:=\frac{1}{2} \int_{\Omega}|\nabla u(x)|^{2} d x,
$$

2000 Mathematics Subject Classification. 34A15, 34K15.

Key words and phrases. Dirichlet energy problem, variational methods, measure preserving maps. 
over the space of admissible maps

$$
\mathcal{A}(\Omega):=\left\{u \in W_{\varphi}^{1,2}\left(\Omega, \mathbb{R}^{n}\right): \operatorname{det} \nabla u=1 \mathcal{L}^{n} \text {-a.e. in } \Omega\right\},
$$

where

$$
W_{\varphi}^{1,2}\left(\Omega, \mathbb{R}^{n}\right)=\left\{u \in W^{1,2}\left(\Omega, \mathbb{R}^{n}\right):\left.u\right|_{\partial \Omega}=\varphi\right\},
$$

and $\varphi$ denotes the identity map. In this paper we are primarily concerned with the problem of extremising the energy functional (1.1) over the space (1.2) and examining a particular class of maps as solutions to the corresponding system of Euler-Lagrange equations (see Section 2)

$$
\begin{cases}\operatorname{div} \mathfrak{S}[x, \nabla u(x)]=0 & \text { for } x \in \Omega, \\ \operatorname{det} \nabla u(x)=1 & \text { for } x \in \Omega, \\ u(x)=\varphi(x) & \text { for } x \in \partial \Omega .\end{cases}
$$

Here, we have that

$$
\mathfrak{S}[x, \mathbf{F}]=\mathbf{F}-\mathfrak{p}(x) \mathbf{F}^{-t}=: \mathfrak{T}[x, \mathbf{F}] \mathbf{F}^{-t},
$$

for $x \in \Omega, \mathbf{F} \in \mathbb{R}^{n \times n}$ satisfying $\operatorname{det} \mathbf{F}=1$ and $\mathfrak{p}$ a suitable Lagrange multiplier while

$$
\mathfrak{T}[x, \mathbf{F}]=\mathbf{F F}^{t}-\mathfrak{p}(x) \mathbf{I} .
$$

A motivating source for this type of problem is nonlinear elasticity where (1.1) and (1.2) represent a simple model of a homogeneous incompressible hyperelastic material and solutions to the above system of equations serve as the corresponding equilibrium states (cf. e.g. Ball [1]). ${ }^{1}$ )

While the question of existence of minimizers for $\mathbb{F}$ (with arbitrary boundary conditions $\varphi$ ) is settled in [1], the regularity of such minimizers and more generally, solutions to the corresponding Euler-Lagrange equations remains at large an outstanding open problem (cf. e.g. [2], [6] and [7]). Furthermore, little is known about the structure of the solution set (e.g. uniqueness or multiplicity, existence of local minimizers, etc.).

For the purpose of this paper we concentrate mainly on the case where the domain $\Omega$ is an $n$-dimensional annulus, that is, $\Omega=\left\{x \in \mathbb{R}^{n}: a<|x|<b\right\}$ with $b>a>0$ and aim to present examples of multiple local minimizers of $\mathbb{F}$ over $\mathcal{A}(\Omega)$, as well as solutions to the corresponding Euler-Lagrange equations. This will be achieved by developing a number of topological tools, most prominently, a notion of degree on the space of self-maps of annuli $\mathfrak{A}$. (See Section 8). Indeed, we show that the latter, when equipped with the topology of uniform

$\left({ }^{1}\right)$ In the language of elasticity, the tensor fields (1.3) and (1.4) are referred to as the Piola-Kirchhoff and the Cauchy stress tensors respectively. 
convergence, consists of infinitely many components when $n=2$ and precisely two when $n \geq 3$. $\left({ }^{2}\right)$

In the case $n=2$, taking advantage of the embedding of $\mathcal{A}(\Omega)$ into $\mathfrak{A}$ enables one to partition $\mathcal{A}(\Omega)$ into a corresponding collection of infinitely many pairwise disjoint sequentially weakly closed subsets on each of which minimizing the Dirichlet energy will give rise to a local minimizer (with respect to the $L^{1}$ metric). On the other hand, by considering the Euler-Lagrange equations separately, one can show by direct verification that the latter admits solutions amongst a class of maps referred to as twists. Interestingly, here, the corresponding equations can be solved completely leading to explicit solutions (depending on $a, b$, as well as $k$, the twist parameter). Furthermore, these twist solutions will be the unique minimizers of the Dirichlet energy, restricted to the class of twists from within a fixed component of $\mathfrak{A}$.

The explicit form of these solutions enables us to further investigate their qualitative properties; one particular question being their limiting behaviour as the inner radius of the annulus converges to zero, i.e. when the inner hole shrinks to a point. This is particularly interesting as in the limit (a punctured disk) all components of the corresponding function space collapse to a single one and so it is important to have a proper understanding of the limiting behaviour of the latter solutions (on each fixed component) together with their corresponding energies. $\left({ }^{3}\right)$

The case $n=3$ is more complicated and less clear as here $\mathcal{A}(\Omega)$ does not embedd into $\mathfrak{A}$ and so there is no similar partitioning of $\mathcal{A}(\Omega)$ available. However, by analogy with the first part it is natural to seek solutions to the Euler-Lagrange equations in the form of higher dimensional twists. Motivated by the results in the case $n=2$ we begin by restricting the Dirichlet energy to the space of twists. It then follows that there exists a unique minimizer corresponding to every twist parameter $k$. Moreover, the latter minimizers, depending on whether $k$ is even or odd lie within the same component of $\mathfrak{A}$. However, interestingly, it will turn out that higher dimensional twists do not form solutions to the full Euler-Lagrange equations, unless when $k=0$. This, above all shows that unlike the case $n=2$, in higher dimensions, minimizing the Dirichlet energy on the space of twists and verifying the resulting Euler-Lagrange equation does not grant a solution to the full Euler-Lagrange equations.

$\left({ }^{2}\right)$ Here, we have aimed at a modest contribution by restricting to the case of annuli. For an arbitrary bounded Lipschitz domain in $\mathbb{R}^{2}$, a characterisation of the components of the space of self-maps of $\Omega$ is given in [12].

$\left({ }^{3}\right)$ In the case of a punctured disk, say, $\Omega=\mathbb{B} \backslash\{0\}$, for any pair of maps $\phi_{0}, \phi_{1} \in \mathfrak{A}:=$ $\{\phi \in C(\bar{\Omega}, \bar{\Omega}): \phi=\varphi$ on $\partial \Omega=\{0\} \cup \partial \mathbb{B}\}$, the path $[0,1] \ni t \mapsto \phi_{t}:=(1-t) \phi_{0}+t \phi_{1}$ lies within $\mathfrak{A}$ and joins $\phi_{0}$ to $\phi_{1}$. 
Finally, we note that the non-existence results, here, are in sharp contrast to the case where the Dirichlet energy is replaced by the so-called $p$-energy

$$
\mathbb{F}_{p}[u, \Omega]:=\frac{1}{p} \int_{\Omega}|\nabla u(x)|^{p} d x,
$$

(with $p \geq n$ ) and over the space of admissible maps

$$
\mathcal{A}_{p}(\Omega):=\left\{u \in W_{\varphi}^{1, p}\left(\Omega, \mathbb{R}^{n}\right): \operatorname{det} \nabla u=1 \mathcal{L}^{n} \text {-a.e. in } \Omega\right\}
$$

as similar arguments lead to, e.g. the existence of multiple local minimizers and hence (potential) solutions to the corresponding Euler-Lagrange equations. (See Remark 7.3.)

\section{The Euler-Lagrange equations}

The derivation of the Euler-Lagrange equations can be facilitated by the use of the Lagrange multiplier method. To this end, let $\mathfrak{p}=\mathfrak{p}(x)$ denote the Lagrange multiplier and consider the unconstrained functional $\left({ }^{4}\right)$

$$
\mathbb{J}[u, \Omega]:=\int_{\Omega}\left[\frac{1}{2}|\nabla u|^{2}-\mathfrak{p}(x)[\operatorname{det} \nabla u-1]\right] d x .
$$

Then, proceeding formally, it is a straight-forward matter to verify that the Euler-Lagrange equations take the form

$$
\int_{\Omega} \sum_{i, j=1}^{n}\left[\frac{\partial u_{i}}{\partial x_{j}}-\mathfrak{p}(x)[\operatorname{cof} \nabla u]_{i j}\right] \frac{\partial \phi_{i}}{\partial x_{j}} d x=0,
$$

for all $\phi \in C_{0}^{\infty}\left(\Omega, \mathbb{R}^{n}\right)$, that is,

$$
\sum_{j=1}^{n} \frac{\partial}{\partial x_{j}}\left[\frac{\partial u_{i}}{\partial x_{j}}-\mathfrak{p}(x)[\operatorname{cof} \nabla u]_{i j}\right]=0
$$

for $1 \leq i \leq n$. In addition with the aid of the so-called Piola identity the latter can be re-written as

$$
\sum_{j=1}^{n}\left[\frac{\partial^{2} u_{i}}{\partial x_{j}^{2}}-[\operatorname{cof} \nabla u]_{i j} \frac{\partial \mathfrak{p}}{\partial x_{j}}\right]=0
$$

$\left({ }^{4}\right)$ In contrast to the Dirichlet energy, the unconstrained functional $\mathbb{J}$ is not well-defined over $W^{1,2}\left(\Omega, \mathbb{R}^{n}\right)$. This is firstly due to the presence of the Lagrange multiplier $\mathfrak{p}$ which is apriori assumed only measurable and secondly the failure of integrability of $\operatorname{det} \nabla u$. The natural way to overcome this is to instead restrict $\mathbb{J}$ to the space

$$
\mathcal{A}_{p, q}(\Omega):=\left\{u \in W_{\varphi}^{1, p}\left(\Omega, \mathbb{R}^{n}\right): \operatorname{cof} \nabla u \in L^{q}\left(\Omega, \mathbb{R}^{n \times n}\right)\right\}
$$

with $p \geq n-1, q \geq n /(n-1)$ and $\mathfrak{p} \in \operatorname{BMO}(\Omega)$. (When $n=2, u \in W^{1,2}\left(\Omega, \mathbb{R}^{2}\right)$ and $\mathfrak{p} \in \operatorname{BMO}(\Omega)$.) However, as in this section we will be mainly concerned with classical solutions to the Euler-Lagrange equations associated with $\mathbb{J}$ (see Definition 2.1) the specific choice of the function spaces will be of no immediate significance. 
or alternatively in vector notation as

$$
\Delta u-[\operatorname{cof} \nabla u] \nabla \mathfrak{p}=0
$$

Furthermore, in view of the trivial identity $\operatorname{det}[\operatorname{cof} \mathbf{F}]=[\operatorname{det} \mathbf{F}]^{n-1}$, it follows from $\operatorname{det} \nabla u=1 \mathcal{L}^{n}$-almost everywhere in $\Omega$ that $\operatorname{det}[\operatorname{cof} \nabla u]=1 \mathcal{L}^{n}$-almost eeverywhere in $\Omega$ and so

$$
[\operatorname{cof} \nabla u]^{-1} \Delta u-\nabla \mathfrak{p}=0
$$

Finally, recalling that $(\operatorname{det} \mathbf{F}) \mathbf{F}^{-1}=[\operatorname{cof} \mathbf{F}]^{t}$, we can re-write this last equation as

$$
[\nabla u]^{t} \Delta u-\nabla \mathfrak{p}=0
$$

Definition 2.1 (Classical solution). A pair $(u, \mathfrak{p})$ is said to be a classical solution to the Euler-Lagrange equations associated with the Dirichlet functional (1.1) and subject to the constraint (1.2) if and only if

(a) $u \in C^{2}\left(\Omega, \mathbb{R}^{n}\right) \cap C\left(\bar{\Omega}, \mathbb{R}^{n}\right)$,

(b) $\mathfrak{p} \in C^{1}(\Omega) \cap C(\bar{\Omega})$, and

(c) $(u, \mathfrak{p})$ satisfy the system of equations

$$
\begin{cases}{[\operatorname{cof} \nabla u(x)]^{-1} \Delta u(x)=\nabla \mathfrak{p}(x)} & \text { for } x \in \Omega, \\ \operatorname{det} \nabla u(x)=1 & \text { for } x \in \Omega, \\ u(x)=x & \text { for } x \in \partial \Omega .\end{cases}
$$

We next discuss an invariance property associated with the unconstrained functional $\mathbb{J}$ along with its Euler-Lagrange equations (2.2). To this end, let $\mathbf{F}$ be a conformal or an anti-conformal matrix, that is, $\mathbf{F}=\kappa \mathbf{Q}$ for some non-zero $\kappa \in \mathbb{R}$ and $\mathbf{Q} \in \mathbf{O}(n)$ the orthogonal group. Then, it is evident that

$$
u \in \mathcal{A}(\Omega) \Leftrightarrow v:=\mathbf{F}^{-1} u \circ \mathbf{F} \in \mathcal{A}\left(\mathbf{F}^{-1} \Omega\right)
$$

Moreover, a straight-forward calculation gives,

$$
\begin{aligned}
\mathbb{J}\left[v, \mathbf{F}^{-1} \Omega\right] & =\int_{\mathbf{F}^{-1} \Omega}\left[\frac{1}{2}|\nabla v|^{2}-p(y)[\operatorname{det} \nabla v-1]\right] d y \\
& =\int_{\mathbf{F}^{-1} \Omega}\left[\frac{1}{2}\left|\mathbf{F}^{-1}[\nabla u] \mathbf{F}\right|^{2}-p(y)\left[\operatorname{det}\left(\mathbf{F}^{-1}[\nabla u] \mathbf{F}\right)-1\right]\right] d y,
\end{aligned}
$$

where $\nabla u=\nabla u(\mathbf{F} y)$. Therefore, making note of the identity

$$
\operatorname{det}\left(\mathbf{F}^{-1}[\nabla u] \mathbf{F}\right)=\operatorname{det} \nabla u
$$


along with $\left({ }^{5}\right)$

$$
\begin{aligned}
\left|\mathbf{F}^{-1}[\nabla u] \mathbf{F}\right|^{2} & =\operatorname{Tr}\left\{\left(\mathbf{F}^{-1}[\nabla u] \mathbf{F}\right)\left(\mathbf{F}^{t}[\nabla u]^{t} \mathbf{F}^{-t}\right)\right\} \\
& =\kappa^{2} \operatorname{Tr}\left\{\left(\mathbf{F}^{-1}[\nabla u]\right)\left([\nabla u]^{t} \mathbf{F}^{-t}\right)\right\} \\
& =\kappa^{2} \operatorname{Tr}\left\{\left([\nabla u]^{t} \mathbf{F}^{-t}\right)\left(\mathbf{F}^{-1}[\nabla u]\right)\right\}=|\nabla u|^{2},
\end{aligned}
$$

we are lead, after the change of variables $x=\mathbf{F} y$, to the energy identity

$$
\begin{aligned}
\mathbb{J}\left[v, \mathbf{F}^{-1} \Omega\right] & =\int_{\mathbf{F}^{-1} \Omega}\left[\frac{1}{2}|\nabla u|^{2}-\mathfrak{p}(y)[\nabla u-1]\right] d y \\
& =\int_{\Omega}\left[\frac{1}{2}|\nabla u|^{2}-\mathfrak{p}(x)[\operatorname{det} \nabla u-1]\right] \frac{1}{\operatorname{det} \mathbf{F}} d x=\frac{1}{\operatorname{det} \mathbf{F}} \mathbb{J}[u, \Omega] .
\end{aligned}
$$

The same argument can also be used to show that for any $\phi \in C_{0}^{\infty}\left(\Omega, \mathbb{R}^{n}\right)$, setting $\psi:=\mathbf{F}^{-1} \phi \circ \mathbf{F} \in C_{0}^{\infty}\left(\mathbf{F}^{-1} \Omega, \mathbb{R}^{n}\right)$ we have

$$
\left.\frac{1}{\operatorname{det} \mathbf{F}} \frac{d}{d t} \mathbb{J}[u+t \phi, \Omega]\right|_{t=0}=\left.\frac{d}{d t} \mathbb{J}\left[v+t \psi, \mathbf{F}^{-1} \Omega\right]\right|_{t=0},
$$

and so we are lead to the following statement.

Proposition 2.2. Let $(u, \mathfrak{p})$ be a solution pair to the Euler-Lagrange equations $(2.2)$ on $\Omega$. Then $(v, \mathfrak{p}):=\left(\mathbf{F}^{-1} u \circ \mathbf{F}, \mathfrak{p} \circ \mathbf{F}\right)$ is a solution pair to (2.2) on $\mathbf{F}^{-1} \Omega$.

\section{A homotopy characterisation of maps in $\mathcal{A}(\Omega)$ when $\Omega \subset \mathbb{R}^{2}$ is an annulus}

Let $\Omega=\left\{x \in \mathbb{R}^{2}: a<|x|<b\right\}$ with $b>a>0$ and consider the space $\left({ }^{6}\right)$

$$
\mathfrak{A}:=\{\phi \in C(\bar{\Omega}, \bar{\Omega}): \phi(x)=x \text { for } x \in \partial \Omega\},
$$

equipped with the topology of uniform convergence. Then, it can be shown that the homotopy classes of $\mathfrak{A}$ can be enumerated by the integers $\mathbb{Z}$. Indeed, for each homotopy class the latter integer can be taken as the index of the curve resulting from restricting any arbitrary representative $\phi$ of the class to a radial ray whilst normalising it so that its range lies on the unit circle. In what follows, this correspondence will be denoted by

$$
[\phi] \mapsto \operatorname{deg}\left(\frac{\phi}{|\phi|}\right) .
$$

$\left(^{5}\right)$ Note that it is only in concluding (2.7) that the assumption of $\mathbf{F}$ being conformal or anti-conformal is used.

$\left({ }^{6}\right)$ The reader is referred to the Appendix for further discussion on the basic properties of the homotoopy classes of $\mathfrak{A}$. 
Since $\Omega \subset \mathbb{R}^{2}$, every $u \in \mathcal{A}:=\mathcal{A}(\Omega)$ has a representative (again, denoted $u$ ) in $\mathfrak{A}$. Hence we can set for each $k \in \mathbb{Z}$,

$$
\mathcal{A}_{k}:=\left\{u \in \mathcal{A}: \operatorname{deg}\left(\frac{u}{|u|}\right)=k\right\} .
$$

Then as a result the latter are pairwise disjoint and that

$$
\mathcal{A}=\bigcup_{k \in \mathbb{Z}} \mathcal{A}_{k}
$$

Proposition 3.1. Let $\Omega=\left\{x \in \mathbb{R}^{2}: a<|x|<b\right\}$ and for $k \in \mathbb{Z}$ consider the classes $\mathcal{A}_{k}$ as defined by (3.1). Then,

(a) $\mathcal{A}_{k}$ is $W^{1,2}$-sequentially weakly closed,

(b) for $u \in \mathcal{A}_{k}$ and $s>0$ there exists $\delta=\delta(u, s)>0$ such that

$$
\text { if } v \in \mathcal{A},\|v-u\|_{L^{1}}<\delta \text { and } \mathbb{F}[v, \Omega]<s \text { then } v \in \mathcal{A}_{k} .
$$

Proof. (a) Let $\left(u_{j}\right)_{j \in \mathbb{N}} \subset \mathcal{A}_{k}$ and $u_{j} \rightarrow u$ in $W^{1,2}\left(\Omega, \mathbb{R}^{2}\right)$. Then, in view of

$$
\operatorname{det} \nabla u_{j} \stackrel{*}{\rightarrow} \operatorname{det} \nabla u
$$

in $\mathcal{M}(\Omega), u \in \mathcal{A}$. Moreover, in view of $u_{j} \rightarrow u$ uniformly on $\bar{\Omega}$, an application of Proposition 8.5 gives $u \in \mathcal{A}_{k}$. This justifies (a).

(b) Assume the contrary. Then, there exist $u \in \mathcal{A}_{k}, s>0$ and $\left(v_{j}\right)_{j \in \mathbb{N}}$ such that

$$
v_{j} \in \mathcal{A}, \quad\left\|v_{j}-u\right\|_{L^{1}} \rightarrow 0 \quad \text { and } \quad \mathbb{F}\left[v_{j}, \Omega\right]<s,
$$

while $v_{j} \notin \mathcal{A}_{k}$. However, the above imply that by passing to a subsequence (we do not re-label this) $v_{j} \rightarrow u$ in $W^{1,2}\left(\Omega, \mathbb{R}^{2}\right)$ and as in (a), $v_{j} \rightarrow u$ uniformly on $\bar{\Omega}$. Hence, again by Proposition 8.5 ,

$$
\operatorname{deg}\left(\frac{v_{j}}{\left|v_{j}\right|}\right) \rightarrow \operatorname{deg}\left(\frac{u}{|u|}\right) .
$$

As the above quantities are integers (with the one on the right being $k$ ), it follows that for $j$ large enough, $v_{j} \in \mathcal{A}_{k}$ which is a contradiction.

In view of the sequential weak lower semicontinuity of the Dirichlet energy, an application of the direct methods of the calculus of variations leads us to the following conclusion.

Proposition 3.2. Let $\Omega=\left\{x \in \mathbb{R}^{2}: a<|x|<b\right\}$ and for $k \in \mathbb{Z}$ consider the classes $\mathcal{A}_{k}$ as defined by (3.1). Then there exists $\bar{u}=\bar{u}(k, a, b, x) \in \mathcal{A}_{k}$ so that

$$
\mathbb{F}[\bar{u}, \Omega]=\inf _{\mathcal{A}_{k}} \mathbb{F}[\cdot, \Omega] .
$$

In addition for each such $\bar{u}$ there exists $\delta=\delta(\bar{u})>0$ such that

$$
\mathbb{F}[\bar{u}, \Omega] \leq \mathbb{F}[v, \Omega]
$$


for all $v \in \mathcal{A}$ satisfying $\|\bar{u}-v\|_{L^{1}}<\delta$.

Proof. Let $\left(v_{j}\right) \subset \mathcal{A}_{k}$ be an infimizing sequence, i.e. $\mathbb{F}\left[v_{j}\right] \downarrow \alpha:=\inf _{\mathcal{A}_{k}} \mathbb{F}<$ $\infty$. Then by passing to a subsequence (not re-labeled) $v_{j} \rightarrow \bar{u}$ in $W^{1,2}\left(\Omega, \mathbb{R}^{2}\right)$ where by (a) in Proposition 3.1, $\bar{u} \in \mathcal{A}_{k}$. Thus,

$$
\alpha \leq \mathbb{F}[\bar{u}, \Omega] \leq \liminf _{j \uparrow \infty} \mathbb{F}\left[v_{j}, \Omega\right] \leq \alpha
$$

and so $u$ is a minimizer as required.

To establish the final assertion, fix $k$ and $\bar{u}$ as above and with $s=1+\mathbb{F}[\bar{u}, \Omega]$ pick $\delta>0$ as (b) in Proposition 3.1. Then, any $v \in \mathcal{A}$ satisfying $\|\bar{u}-v\|_{L^{1}}<\delta$ also satisfies (3.2) (as otherwise $\mathbb{F}[v, \Omega]<\mathbb{F}[\bar{u}, \Omega]<s$ implying that $v \in \mathcal{A}_{k}$ and hence in view of $\bar{u}$ being a minimizer, $\mathbb{F}[v, \Omega] \geq \mathbb{F}[\bar{u}, \Omega]$ which is a contradiction)

\section{Twists as solutions in two dimensions}

When $n=2$ the Euler-Lagrange equations associated with the Dirichlet energy $\mathbb{F}$ over the space $\mathcal{A}(\Omega)$ take the form

$$
\left[\begin{array}{c}
\Delta u_{1} \\
\Delta u_{2}
\end{array}\right]=\left[\begin{array}{cc}
u_{2,2} & -u_{2,1} \\
-u_{1,2} & u_{1,1}
\end{array}\right]\left[\begin{array}{l}
\mathfrak{p}_{, 1} \\
\mathfrak{p}_{, 2}
\end{array}\right]
$$

or equivalently in view of the $\mathcal{L}^{2}$-a.e. invertibility of the matrix $[\operatorname{cof} \nabla u]$ in $\Omega$ that indeed

$$
\left[\begin{array}{l}
\mathfrak{p}_{, 1} \\
\mathfrak{p}_{, 2}
\end{array}\right]=\left[\begin{array}{ll}
u_{1,1} & u_{2,1} \\
u_{1,2} & u_{2,2}
\end{array}\right]\left[\begin{array}{l}
\Delta u_{1} \\
\Delta u_{2}
\end{array}\right]
$$

In this section we consider the case where $\Omega \subset \mathbb{R}^{2}$ is a two dimensional annulus and discuss a particular class of maps, namely, twists that will eventually be shown to give rise to classical solutions to the Euler-Lagrange equations (4.1) or $(4.2) .\left({ }^{7}\right)$

Definition 4.1 (Twist). Let $\Omega=\left\{x \in \mathbb{R}^{2}: a<|x|<b\right\}$. A map $u \in$ $C(\bar{\Omega}, \bar{\Omega})$ is referred to as a twist if and only if it can be represented in polar coordinates as $u:(r, \theta) \mapsto(r, \theta+g(r))$.

The function $g$ in the above is referred to as the corresponding twist function. Evidently if $u$ is a twist then $u=\left(u_{1}, u_{2}\right)=(r \cos \omega, r \sin \omega)$ where $\omega=\theta+g(r)$.

Proposition 4.2. Let $\Omega=\left\{x \in \mathbb{R}^{2}: a<|x|<b\right\}$. A twist $u$ lies in $\mathcal{A}=$ $\mathcal{A}(\Omega)$ provided that the corresponding twist function $g$ satisfies the followings:

(a) $g \in W^{1,2}(a, b)$,

(b) $g(a)=2 \pi n_{a}$ for some $n_{a} \in \mathbb{Z}$,

(c) $g(b)=2 \pi n_{b}$ for some $n_{b} \in \mathbb{Z}$.

$\left({ }^{7}\right)$ Twists play a central role in the study of mapping class groups of 2-manifolds and our terminology is motivated by the latter (see e.g. [5]). 
Furthermore, $u \in \mathcal{A}_{k}$ provided that, additionally,

(d) $n_{b}-n_{a}=k$.

Proof. If $u$ is a twist, then $u=\left(u_{1}, u_{2}\right)=(r \cos \omega, r \sin \omega)$ where $\omega=$ $\theta+g(r)$. Thus, from (b) and (c) it follows that $\left.u\right|_{\partial \Omega}=x$. Moreover, a straightforward calculation gives

$$
\begin{aligned}
\nabla u=\left[\begin{array}{ll}
u_{1,1} & u_{1,2} \\
u_{2,1} & u_{2,2}
\end{array}\right] & =\left[\begin{array}{cc}
\cos g-r g^{\prime} \sin \omega \cos \theta & -\sin g-r g^{\prime} \sin \omega \sin \theta \\
\sin g+r g^{\prime} \cos \omega \cos \theta & \cos g+r g^{\prime} \cos \omega \sin \theta
\end{array}\right] \\
& =\left[\begin{array}{cc}
\cos g & -\sin g \\
\sin g & \cos g
\end{array}\right]+r g^{\prime}\left[\begin{array}{c}
-\sin \omega \\
\cos \omega
\end{array}\right] \otimes\left[\begin{array}{c}
\cos \theta \\
\sin \theta
\end{array}\right] .
\end{aligned}
$$

In particular it follows that $\operatorname{det} \nabla u(x)=1$, for all $x \in \Omega$. Next, to justify $u \in W^{1,2}\left(\Omega, \mathbb{R}^{2}\right)$ it is enough to notice that

$$
|\nabla u|^{2}=2+r^{2} g^{\prime 2}(r) .
$$

Thus in view of $|u|=r$ we have that

$$
\int_{\Omega}|u|^{2}+|\nabla u|^{2}=\int_{0}^{2 \pi} \int_{a}^{b}\left[2+r^{2}\left(1+g^{\prime 2}\right)\right] r d r d \theta=2 \pi \int_{a}^{b}\left[2+r^{2}\left(1+g^{\prime 2}\right)\right] r d r
$$

and so referring to (a) the conclusion follows. Finally,

$$
\operatorname{deg}\left(\frac{u}{|u|}\right)=\frac{1}{2 \pi i} \int_{u /|u|} \frac{d z}{z}=\frac{1}{2 \pi} \int_{a}^{b} g^{\prime}(r) d r=n_{b}-n_{a} .
$$

The proof is thus complete.

REMARK 4.3. Adding any integer multiple of $2 \pi$ to $g$ does not affect $u$. Therefore in what follows when speaking of a twist $u$ we agree to set $g(a)=0$ while $g(b)=2 \pi k:=2 \pi\left(n_{b}-n_{a}\right)$.

We now seek solutions to the Euler-Lagrange equations (4.2) in the form of twists. Indeed a straight-forward calculation gives

$$
\Delta u=\left[\begin{array}{l}
\Delta u_{1} \\
\Delta u_{2}
\end{array}\right]=\left[\begin{array}{c}
-3 g^{\prime} \sin \omega-r g^{\prime \prime} \sin \omega-r g^{2} \cos \omega \\
3 g^{\prime} \cos \omega+r g^{\prime \prime} \cos \omega-r g^{2} \sin \omega
\end{array}\right] .
$$

Hence upon substitution we have

$$
\begin{aligned}
{\left[\begin{array}{l}
\mathfrak{p}_{, 1} \\
\mathfrak{p}_{, 2}
\end{array}\right]=} & {\left[\begin{array}{cc}
\cos g-r g^{\prime} \sin \omega \cos \theta & \sin g+r g^{\prime} \cos \omega \cos \theta \\
-\sin g-r g^{\prime} \sin \omega \sin \theta & \cos g+r g^{\prime} \cos \omega \sin \theta
\end{array}\right] } \\
& \times\left[\begin{array}{c}
-3 g^{\prime} \sin \omega-r g^{\prime \prime} \sin \omega-r g^{\prime 2} \cos \omega \\
3 g^{\prime} \cos \omega+r g^{\prime \prime} \cos \omega-r g^{\prime 2} \sin \omega
\end{array}\right] \\
= & {\left[\begin{array}{c}
-r g^{\prime 2} \cos \theta+\left(3 g^{\prime}+r g^{\prime \prime}\right)\left(r g^{\prime} \cos \theta-\sin \theta\right) \\
-r g^{\prime 2} \sin \theta+\left(3 g^{\prime}+r g^{\prime \prime}\right)\left(r g^{\prime} \sin \theta+\cos \theta\right)
\end{array}\right] }
\end{aligned}
$$

or alternatively that

$$
\left[\begin{array}{c}
\mathfrak{p}_{, r} \\
\mathfrak{p}_{, \theta}
\end{array}\right]=\left[\begin{array}{c}
r g^{\prime}\left(2 g^{\prime}+r g^{\prime \prime}\right) \\
r\left(3 g^{\prime}+r g^{\prime \prime}\right)
\end{array}\right]
$$


As a necessary condition for the solvability of (4.3) is for the right-hand side to be curl-free we arrive at $\left(^{8}\right)$

$$
0=\frac{\partial}{\partial r}\left[r\left(3 g^{\prime}+r g^{\prime \prime}\right)\right]-\frac{\partial}{\partial \theta}\left[r g^{\prime}\left(2 g^{\prime}+r g^{\prime \prime}\right)\right]=r^{2}\left[g^{\prime \prime \prime}+\frac{5}{r} g^{\prime \prime}+\frac{3}{r^{2}} g^{\prime}\right]
$$

It is plain that (4.4) is an Euler-type equation and admits solutions in the form $g(r)=r^{\alpha}$. This upon substitution gives $\alpha \in\{0,-2\}$ [with $\alpha=0$ repeated]. Hence $g(r)=c_{1}+c_{2} \ln r+c_{3} r^{-2}$. However (4.3) being a gradient demands $c_{2}=0$. Finally, using the boundary conditions $g(a)=0$ and $g(b)=2 k \pi$ we arrive at $\left({ }^{9}\right)$

$$
g(r)=2 k \pi \frac{a^{2} b^{2}}{b^{2}-a^{2}}\left[\frac{1}{a^{2}}-\frac{1}{r^{2}}\right] .
$$

Proposition 4.4. Let $\Omega=\left\{x \in \mathbb{R}^{2}: a<|x|<b\right\}$. Then, for each $k \in \mathbb{Z}$, the Euler-Lagrange equations associated with $\mathbb{F}$ have a solution $u \in \mathcal{A}_{k}$ in the form of a twist where

$$
u=u(k, a, b, x):(r, \theta) \mapsto(r, \theta+g(k, a, b, r))
$$

with g given by (4.5).

REMARK 4.5. The above calculation also shows that the Lagrange multiplier $\mathfrak{p}$ is such that

$$
\nabla \mathfrak{p}(x)=-16 k^{2} \pi^{2} \frac{a^{4} b^{4}}{\left(b^{2}-a^{2}\right)^{2}} \frac{x}{|x|^{6}},
$$

which fixes $\mathfrak{p}$ up to an additive constant, that is,

$$
\mathfrak{p}(x)=\mathfrak{p}_{0}+\left[2 k \pi \frac{a^{2} b^{2}}{b^{2}-a^{2}}\right]^{2} \frac{1}{|x|^{4}} .
$$

It can thus be seen that the pair $(u, \mathfrak{p})$ is smooth and so in particular a classical solution (see Definition 2.1).

REMARK 4.6. A classical result in complex function theory asserts that a pair of annuli are conformally equivalent if and only if the ratio of their outer radii to inner radii is a fixed constant, in which case the conformal mapping is given by a linear map. (See e.g. [11, pp. 291-293].) In conjunction with Proposition 2.2 this shows that the solution pairs $(u, \mathfrak{p})$ on the collection of annuli are in fact conformally invariant.

$\left({ }^{8}\right)$ It is quite remarkable that the nonlinear equations (4.2) in the unknown $u$ have now been transformed into a completely linear equation in $g$.

$\left({ }^{9}\right)$ Naturally, here, by increasing the order of the equation, we may introduce additional solutions and so it is crucial that once the general solutions to (4.3) are found we return and eliminate the latter by imposing the requirement that the resulting field on the right in (4.3) be a gradient. 
Note finally that for a twist $u$ with the corresponding twist function $g$ as in (4.5) the Dirichlet energy is given by

$$
\mathbb{F}[u, \Omega]=\pi\left[\left(b^{2}-a^{2}\right)+8 k^{2} \pi^{2} \frac{a^{2} b^{2}}{b^{2}-a^{2}}\right] .
$$

\section{Minimizing properties of the twist solutions}

We now proceed by considering the minimizing properties of the twist solutions obtained through Proposition 4.4. Indeed, for any twist $u$ we have that

$$
\frac{1}{2} \int_{\Omega}|\nabla u|^{2}=\int_{0}^{2 \pi} \int_{a}^{b}\left[1+\frac{1}{2} r^{2} g^{\prime 2}\right] r d r d \theta=2 \pi \int_{a}^{b}\left[1+\frac{1}{2} r^{2} g^{\prime 2}\right] r d r .
$$

Motivated by this, let

$$
\mathbb{E}[g]:=\int_{a}^{b}\left[1+\frac{1}{2} r^{2} g^{\prime 2}\right] r d r
$$

and for $k \in \mathbb{Z}$ put

$$
\mathcal{E}_{k}:=\left\{g \in W^{1,2}(a, b): g(a)=0, g(b)=2 \pi k\right\}
$$

It is a trivial matter to verify that the Euler-Lagrange equation corresponding to $\mathbb{E}$ takes the form,

$$
\frac{d}{d r}\left[r^{3} g^{\prime}(r)\right]=0
$$

which together with the boundary conditions $g(a)=0$ and $g(b)=2 k \pi$ admits the unique solution $g$ as given by (4.5). This, together with the convexity of $\mathbb{E}$ immediately leads to the following conclusion.

Proposition 5.1. For each $k \in \mathbb{Z}$, the twist function g given by (4.5) is the unique minimizer of $\mathbb{E}$ over $\mathcal{E}_{k}$.

We now return to the unconstrained energy functional $\mathbb{J}$ as in (2.1). Let $u \in W^{1,2}\left(\Omega, \mathbb{R}^{2}\right)$ and $v \in W_{0}^{1,2}\left(\Omega, \mathbb{R}^{2}\right)$. Then, in view of the quadratic nature of $\mathbb{J}$ when $n=2$, we can write

$$
\begin{aligned}
\mathbb{J}[u+v]-\mathbb{J}[u]= & \int_{\Omega}\left[\frac{1}{2}|\nabla u+\nabla v|^{2}-\mathfrak{p}(x)[\operatorname{det}(\nabla u+\nabla v)-1]\right] d x \\
& -\int_{\Omega}\left[\frac{1}{2}|\nabla u|^{2}-\mathfrak{p}(x)[\operatorname{det} \nabla u-1]\right] d x \\
= & \int_{\Omega}[\nabla u \cdot \nabla v-\mathfrak{p}(x) \operatorname{cof} \nabla u \cdot \nabla v \\
& \left.+\frac{1}{2}|\nabla v|^{2}-\mathfrak{p}(x) \operatorname{det} \nabla v\right] d x
\end{aligned}
$$


Hence, if $u$ is a (weak) solution to the Euler-Lagrange equations (4.1) it follows that

$$
\mathbb{J}[u+v]-\mathbb{J}[u]=\int_{\Omega}\left[\frac{1}{2}|\nabla v|^{2}-\mathfrak{p}(x) \operatorname{det} \nabla v\right] d x .
$$

The following proposition is concerned with the positivity of this quadratic form over $W_{0}^{1,2}\left(\Omega, \mathbb{R}^{2}\right)$ which amounts to $u$ being a minimizer of the unconstrained functional $\mathbb{J}$ over $W_{\phi}^{1,2}\left(\Omega, \mathbb{R}^{2}\right)$.

Proposition 5.2. Let $\Omega \subset \mathbb{R}^{2}$ be a bounded Lipschitz domain. Then there exist $c_{1}, c_{2}>0$ so that if $(u, \mathfrak{p})$ with $u \in \mathcal{A}(\Omega)$ and $\mathfrak{p} \in \mathrm{BMO}(\Omega)$ form a solution pair to (4.1) the followings hold:

(a) if $|\mathfrak{p}|_{\mathrm{BMO}}<c_{1}$ then (5.2) is positive for all non-zero $v \in W_{0}^{1,2}\left(\Omega, \mathbb{R}^{2}\right)$,

(b) if (5.2) is non-negative for all $v \in W_{0}^{1,2}\left(\Omega, \mathbb{R}^{2}\right)$ then $|\mathfrak{p}|_{\mathrm{BMO}}<c_{2}$.

PROOF. (a) In view of the well-known $\mathcal{H}^{1}$-BMO duality we can write

$$
\left|\int_{\Omega} \mathfrak{p}(x) \operatorname{det} \nabla v d x\right| \leq|\mathfrak{p}|_{\mathrm{BMO}}|| \operatorname{det} \nabla v\left\|_{\mathcal{H}^{1}} \leq c|\mathfrak{p}|_{\mathrm{BMO}}|| \nabla v\right\|_{L^{2}}^{2} .
$$

Therefore

$$
\mathbb{J}[u+v]-\mathbb{J}[u]=\int_{\Omega}\left[\frac{1}{2}|\nabla v|^{2}-\mathfrak{p}(x) \operatorname{det} \nabla v\right] d x \geq\left[\frac{1}{2}-c|\mathfrak{p}|_{\mathrm{BMO}}\right] \int_{\Omega}|\nabla v|^{2} d x
$$

and so (a) follows by choosing $c_{1}=(2 c)^{-1}$.

(b) This is a consequence of Theorem 1 in [16] to which the reader is referred.

REMARK 5.3. By utilising the null-Lagrangian structure of the determinant and the trivial bound $|\mathbf{F}|^{2} \geq 2|\operatorname{det} \mathbf{F}|$, it is possible to prove directly (i.e. avoiding the $\mathcal{H}^{1}$-BMO duality] that the quadratic form (5.2) is positive (as (a) in Proposition 5.2), if, there exists $\mathfrak{p}_{0}$ such that

$$
\sup _{x \in \Omega}\left|\mathfrak{p}(x)-\mathfrak{p}_{0}\right|<1 .
$$

Evidently (5.3) is more quantitative than condition (a) in Proposition 5.2, formulated in terms of $c_{1}>0$. (Note also that (5.3) gives $|\mathfrak{p}|_{\mathrm{BMO}}<2$.)

Motivated by the above the next question to address now is if any of the twist solutions $u$ from Proposition 4.4 are indeed minimizers of the unconstrained functional $\mathbb{J} ?\left({ }^{10}\right)$ Indeed, without much extra effort, we can state the following result.

\footnotetext{
$\left({ }^{10}\right)$ It can be easily verified that this need not be the case in certain parameter regimes for $a, b$ and $k$; e.g. (b) in Proposition 5.2 fails for any fixed $a<b$ and large enough $|k|$. (It is also instructive to consider (5.3) when the annulus converges to a ring.)
} 
Proposition 5.4. Let $\Omega=\left\{x \in \mathbb{R}^{2}: a<|x|<b\right\}$. Then, for $k \neq 0$ the twist solution $u=u(k, a, b, x)$ is not a minimizer of the unconstrained functional $\mathbb{J}$.

ProOF. In view of the strict convexity of $\mathbb{F}\left[\right.$ on $\left.W^{1,2}\left(\Omega, \mathbb{R}^{2}\right)\right]$, we have $\mathbb{F}[x]<$ $\mathbb{F}[u]$, unless $u=x$. However, this together with the identity $\mathbb{F}=\mathbb{J}$ on $\mathcal{A}(\Omega)$ implies $\mathbb{J}[x]<\mathbb{J}[u]$, unless $u=x$, that is, $k=0$. Hence, when $k \neq 0$ no $u(k, a, b, x)$ is a minimizer of $\mathbb{J}$.

REMARK 5.5. As a result there is no parameter regime for $a, b$ and $k$ (nonzero) for which the condition (a) in Proposition 5.2 or (5.3) is satisfied!

REMARK 5.6. Though plausible, it remains open if the twist $u=u(k, a, b, x)$ from Proposition 4.4 is indeed a minimizer of $\mathbb{F}$ over $\mathcal{A}_{k}$.

\section{Asymptotic behaviour of twists as the inner hole shrinks to a point}

In this section we consider the case where $b=1$ and $a=\varepsilon>0$ with the aim of discussing the limiting properties of the components $\mathcal{A}_{k}$ as well as the solutions $u_{\varepsilon}:=u(k, \varepsilon, 1, x)$ from Proposition 4.4 as $\varepsilon \downarrow 0$. This is particularly interesting as in the limit (the punctured disk) all components of the function space collapse to a single one and so it is important to have a clear understanding as to how the twist solutions and their energies (for each fixed $k$ ) behave.

To this end, let $\Omega_{\varepsilon}:=\left\{x \in \mathbb{R}^{2}: \varepsilon<|x|<1\right\}$ and for each $k \in \mathbb{Z}$ let $u_{\varepsilon} \in \mathcal{A}_{k}$ denote the twist with the corresponding twist function

$$
g_{\varepsilon}(r)=2 k \pi \frac{\varepsilon^{2}}{1-\varepsilon^{2}}\left[\frac{1}{\varepsilon^{2}}-\frac{1}{r^{2}}\right] .
$$

In order to make the study of the limiting properties of $u_{\varepsilon}$ more tractable, we fix the domain to be the unit disk, and extend each map by identity off $\Omega_{\varepsilon}$. (In what follows unless otherwise stated we speak of $u_{\varepsilon}$ in this extended sense.) Thus here we have that

$$
u_{\varepsilon}:(r, \theta) \mapsto\left(r, \theta+h_{\varepsilon}(r)\right)
$$

where

$$
h_{\varepsilon}(r)= \begin{cases}g_{\varepsilon}(r) & \text { for } \varepsilon \leq r \leq 1 \\ 0 & \text { for } r \leq \varepsilon\end{cases}
$$

Proposition 6.1. The function $h_{\varepsilon}$ enjoys the following properties:

(a) $h_{\varepsilon} \in C[0,1]$,

(b) $h_{\varepsilon}$ is non-decreasing,

(c) $\left|h_{\varepsilon}\right| \leq 2 \pi|k|$ on $[0,1]$,

(d) for $\delta \in(0,1)$ there exist $\varepsilon_{0}>0$ and $\sigma>1$ such that for $\varepsilon \leq \varepsilon_{0}$ we have that $\left|h_{\varepsilon}-2 k \pi\right| \leq \delta$ on $[\sigma \varepsilon, 1]$. 


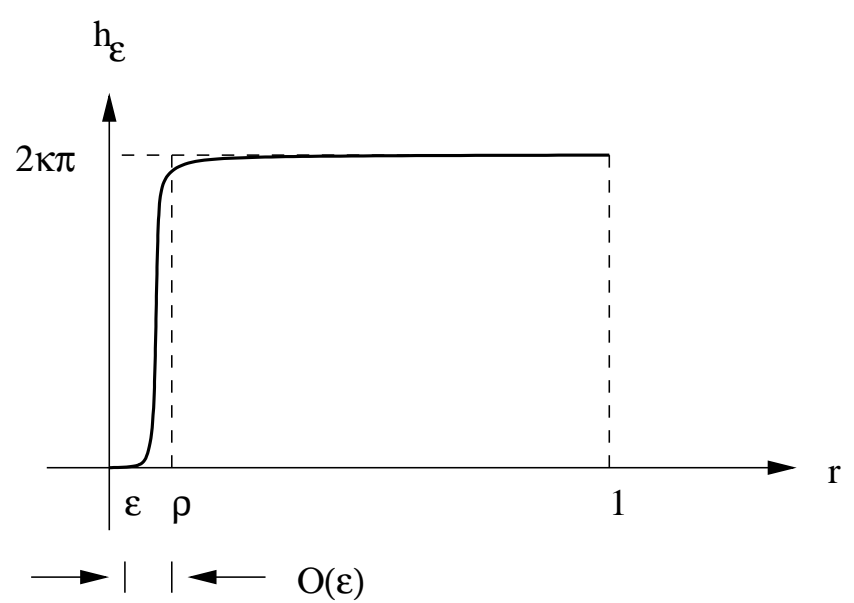

Figure 1. The twist function $h_{\varepsilon}$

Proof. Assertions (a)-(c) are immediate consequences of the definition. To justify (d) we assume $k \neq 0$ (as otherwise the conclusion is trivially true). Now for $r \in[\varepsilon, 1]$ we have

(6.1) $\left|h_{\varepsilon}(r)-2 k \pi\right|=\left|2 k \pi\left[\frac{1}{1-\varepsilon^{2}}\left(1-\frac{\varepsilon^{2}}{r^{2}}\right)-1\right]\right|=2|k| \pi\left(\frac{1}{\varepsilon^{2}}-1\right)^{-1}\left(\frac{1}{r^{2}}-1\right)$.

Set $\sigma=2 \sqrt{|k| \pi \delta^{-1}}$. Then if $\delta<4|k| \pi$ and $\varepsilon<\sqrt{\delta / 4|k| \pi}$ we have $\sigma \varepsilon \in(\varepsilon, 1)$. Hence subject to $\varepsilon_{0}:=\min (\sqrt{\delta / 4|k| \pi}, \sqrt{2} / 2)$ and $r \in[\sigma \varepsilon, 1]$ we can write

$$
\frac{1}{r^{2}}-1 \leq \frac{1}{\sigma^{2} \varepsilon^{2}}-1 \leq \frac{\delta}{2|k| \pi} \frac{1 / 2}{\varepsilon^{2}} \leq \frac{\delta}{2|k| \pi}\left[\frac{1}{\varepsilon^{2}}-1\right]
$$

which together with (6.1) gives the required conclusion.

In view of Proposition 5.1 and the minimizing property of $g_{\varepsilon}$ it is evident that the function

$$
(0,1) \ni \varepsilon \mapsto \mathbb{F}\left[u_{\varepsilon}, \mathbb{B}\right]
$$

is monotone increasing. $\left({ }^{11}\right)$ This, in particular gives the uniform boundedness of the family of solutions $\left(u_{\varepsilon}\right) \subset W^{1,2}\left(\Omega, \mathbb{R}^{2}\right)$ and so we are lead to the following interesting conclusion.

$\left.{ }^{11}\right)$ In fact direct verification based on (4.6) shows the apparently stronger conclusion that for $|k|>0$,

$$
(0,1) \ni \varepsilon \mapsto \mathbb{F}\left[u_{\varepsilon}, \Omega_{\varepsilon}\right]=\mathbb{F}\left[u_{\varepsilon}, \mathbb{B}\right]-\mathbb{F}\left[x, \mathbb{B}_{\varepsilon}\right]
$$

is a monotone increasing function. 
Proposition 6.2. Let $\Omega_{\varepsilon}:=\left\{x \in \mathbb{R}^{2}: \varepsilon<|x|<1\right\}$ and for each $k \in \mathbb{Z}$ let $u_{\varepsilon}:=u(k, \varepsilon, 1, x)$ denote the solution to equations (4.2) from Proposition 4.4. Then:

(a) $u_{\varepsilon} \rightarrow x$ in $W^{1,2}\left(\mathbb{B}, \mathbb{R}^{2}\right)$,

(b) $u_{\varepsilon} \rightarrow x$ uniformly on $\overline{\mathbb{B}}$.

REMARK 6.3. The uniform convergence $u_{\varepsilon} \rightarrow x$ at first looks counterintuitive, as, for $k \neq 0$,

$$
k=\operatorname{deg}\left(\frac{u_{\varepsilon}}{\left|u_{\varepsilon}\right|}\right) \nrightarrow \operatorname{deg}\left(\frac{x}{|x|}\right)=0 .
$$

Stated differently, how can $u_{\varepsilon}$ and $x$ be uniformly close when $u_{\varepsilon}$ twists $k$ times while the limit $u=x$ none?

Proposition 6.1 reveals that the latter twists occur at a distance $\varepsilon$ from the origin and within a layer of thickness $O(\varepsilon)$ (see Figure 1) and this is in no conflict with the stated uniform convergence.

Proof of Proposition 6.2. Fix $k \in \mathbb{Z}$ and consider the family $\left(u_{\varepsilon}\right)$. Then referring to the monotonicity of (6.2) or else using directly the identity

$$
\frac{1}{2} \int_{\mathbb{B}}\left|\nabla u_{\varepsilon}\right|^{2} d x=\pi\left[1+8 k^{2} \pi^{2} \frac{\varepsilon^{2}}{1-\varepsilon^{2}}\right],
$$

it follows that $\left(u_{\varepsilon}\right)$ is uniformly bounded in $W^{1,2}\left(\mathbb{B}, \mathbb{R}^{2}\right)$. Hence by passing to a subsequence (not re-labelled) we have that

$$
\begin{cases}u_{\varepsilon} \rightarrow u & \text { in } W^{1,2}\left(\mathbb{B}, \mathbb{R}^{2}\right), \\ u_{\varepsilon} \rightarrow u & \text { uniformly on } \overline{\mathbb{B}}, \\ \operatorname{det} \nabla u_{\varepsilon} \stackrel{*}{\rightarrow} \operatorname{det} \nabla u & \text { in } \mathcal{M}(\mathbb{B}),\end{cases}
$$

for some $u \in \mathcal{A}$. Thus to complete the proof, we show that $u_{\varepsilon} \rightarrow x$ in $L^{2}\left(\mathbb{B}, \mathbb{R}^{n}\right)$ (the entire family and not just the latter subsequence). Since, for $k=0, u_{\varepsilon}=\mathrm{x}$, in what follows we assume $|k|>0$.

Indeed fix $\delta \in(0,1)$. Then referring to Proposition 6.1 for $\varepsilon<\varepsilon_{0}$ and $\rho:=\sigma \varepsilon=2 \varepsilon \sqrt{|k| \pi \delta^{-1}}$ we can write

$$
\begin{aligned}
\left\|u_{\varepsilon}-x\right\|_{L^{2}}^{2} & =\int_{\mathbb{B}}\left|u_{\varepsilon}(x)-x\right|^{2} d x \\
& =\int_{0}^{2 \pi} \int_{0}^{1} r^{2}|(\cos \omega-\cos \theta, \sin \omega-\sin \theta)|^{2} r d r d \theta \\
& =8 \pi \int_{0}^{1} r^{3} \sin ^{2} \frac{h_{\varepsilon}}{2} d r=8 \pi\left[\int_{\varepsilon}^{\rho}+\int_{\rho}^{1}\right]=\mathrm{I}+\mathrm{II} .
\end{aligned}
$$

However a straight-forward calculation gives

$$
|\mathrm{I}| \leq 8 \pi \int_{\varepsilon}^{\rho} r^{3} d r=2 \pi\left[\frac{2^{4} k^{2} \pi^{2}}{\delta^{2}}-1\right] \varepsilon^{4},
$$


while

$$
|\mathrm{II}| \leq 8 \pi \int_{\rho}^{1} \sin ^{2} \frac{h_{\varepsilon}}{2} d r \leq 2 \pi \delta^{2}
$$

and so the conclusion follows.

Proposition 6.4. Let $\Omega_{\varepsilon}:=\left\{x \in \mathbb{R}^{2}: \varepsilon<|x|<1\right\}$ and for each $k \in \mathbb{Z}$ let $u_{\varepsilon}:=u(k, \varepsilon, 1, x)$ denote the solution to equations (4.2) from Proposition 4.4. Then:

(a) $u_{\varepsilon} \rightarrow x$ in $W^{1,2}\left(\mathbb{B}, \mathbb{R}^{2}\right)$,

(b) $\mathbb{F}\left[u_{\varepsilon}, \mathbb{B}\right] \rightarrow \mathbb{F}[x, \mathbb{B}]$.

Proof. (a) follows from (b), the uniform convexity of the $W^{1,2}$-norm and (a) in Proposition 6.2 , while (b) follows from (6.3) by noting that $\mathbb{F}[x, \mathbb{B}]=\pi$.

Remark 6.5. The convergence $u_{\varepsilon} \rightarrow x$ in $L^{2}\left(\mathbb{B}, \mathbb{R}^{2}\right)$ proved in Proposition 6.2 can be justified alternatively as follows. Indeed, since, $u_{\varepsilon} \rightarrow u$ in $W^{1,2}\left(\mathbb{B}, \mathbb{R}^{2}\right)$, appealing to the $W^{1,2}$-sequential weak lower semicontinuity of $\mathbb{F}$, we have that

$$
\mathbb{F}[x, \mathbb{B}] \leq \mathbb{F}[u, \mathbb{B}] \leq \liminf _{\varepsilon \downarrow 0} \mathbb{F}\left[u_{\varepsilon}, \mathbb{B}\right] .
$$

This, in conjunction with (b) in Proposition 6.4 and the strict convexity of $\mathbb{F}$ (on $W^{1,2}\left(\mathbb{B}, \mathbb{R}^{2}\right)$ ) gives $u=x$. The conclusion now follows from Rellich-Kondrachev compactness theorem.

\section{Twists in higher dimensions}

When $n=3$, the Euler-Lagrange equations associated with the Dirichlet energy $\mathbb{F}$ over the space $\mathcal{A}(\Omega)$ take the form

$$
\left[\begin{array}{l}
\mathfrak{p}_{, 1} \\
\mathfrak{p}_{, 2} \\
\mathfrak{p}_{, 3}
\end{array}\right]=\left[\begin{array}{lll}
u_{1,1} & u_{2,1} & u_{3,1} \\
u_{1,2} & u_{2,2} & u_{3,2} \\
u_{1,3} & u_{2,3} & u_{3,3}
\end{array}\right]\left[\begin{array}{c}
\Delta u_{1} \\
\Delta u_{2} \\
\Delta u_{3}
\end{array}\right] .
$$

In this section, we consider the case where $\Omega \subset \mathbb{R}^{3}$ is a three dimensional annulus and motivated by the analysis in earlier sections discuss three dimensional twists with the intention of finding classical solutions to (7.1) among such maps. $\left({ }^{12}\right)$

Definition 7.1 (Twist). Let $\Omega=\left\{x \in \mathbb{R}^{3}: a<|x|<b\right\}$. A map $u \in$ $C(\bar{\Omega}, \bar{\Omega})$ is referred to as a twist if and only if it can be represented in spherical coordinates as $u:(r, \theta, \phi) \mapsto(r, \theta+g(r), \phi)$.

\footnotetext{
$\left({ }^{12}\right)$ As will be outlined later higher dimensional twists act as generators for homotopy classes of self-maps of annuli and are therefore the natural analogues of their two dimensional counterparts.
} 
Evidently, if $u=\left(u_{1}, u_{2}, u_{3}\right):(r, \theta, \phi) \mapsto(r, \theta+g(r), \phi)$ is a twist, then its components are given through

$$
\left\{\begin{array}{l}
u_{1}=r \sin \phi \cos \omega, \\
u_{2}=r \sin \phi \sin \omega, \\
u_{3}=r \cos \phi,
\end{array}\right.
$$

where $\omega=\theta+g(r)$. Here, similar to the case in two dimensions, we refer to $g$ as the corresponding twist function.

Proposition 7.2. Let $\Omega=\left\{x \in \mathbb{R}^{3}: a<|x|<b\right\}$. A twist $u$ lies in $\mathcal{A}$ provided that the corresponding twist function $g$ satisfies the followings:

(a) $g \in W^{1,2}(a, b)$

(b) $g(a)=2 \pi n_{a}$ for some $n_{a} \in \mathbb{Z}$,

(c) $g(b)=2 \pi n_{b}$ for some $n_{b} \in \mathbb{Z}$.

Proof. From (b) and (c) it follows that $\left.u\right|_{\partial \Omega}=x$. Moreover a straightforward calculation gives

$$
\left[\frac{\partial\left(u_{1}, u_{2}, u_{3}\right)}{\partial(r, \theta, \phi)}\right]=\left[\begin{array}{ccc}
\sin \phi\left(\cos \omega-r g^{\prime} \sin \omega\right) & -r \sin \phi \sin \omega & r \cos \phi \cos \omega \\
\sin \phi\left(\sin \omega+r g^{\prime} \cos \omega\right) & r \sin \phi \cos \omega & r \cos \phi \sin \omega \\
\cos \phi & 0 & -r \sin \phi
\end{array}\right]
$$

In particular we have that

$$
\operatorname{det}\left[\frac{\partial\left(u_{1}, u_{2}, u_{3}\right)}{\partial(r, \theta, \phi)}\right]=-r^{2} \sin \phi
$$

and so

$$
\operatorname{det} \nabla u(x)=\operatorname{det}\left[\frac{\partial\left(u_{1}, u_{2}, u_{3}\right)}{\partial(r, \theta, \phi)}\right] \operatorname{det}\left[\frac{\partial(r, \theta, \phi)}{\partial\left(x_{1}, x_{2}, x_{3}\right)}\right]=1,
$$

for all $x \in \Omega$. In addition we can write

$$
\begin{aligned}
\nabla u= & {\left[\frac{\partial\left(u_{1}, u_{2}, u_{3}\right)}{\partial(r, \theta, \phi)}\right]\left[\frac{\partial(r, \theta, \phi)}{\partial\left(x_{1}, x_{2}, x_{3}\right)}\right] } \\
= & {\left[\begin{array}{ccc}
\sin \phi\left(\cos \omega-r g^{\prime} \sin \omega\right) & -r \sin \phi \sin \omega & r \cos \phi \cos \omega \\
\sin \phi\left(\sin \omega+r g^{\prime} \cos \omega\right) & r \sin \phi \cos \omega & r \cos \phi \sin \omega \\
\cos \phi & 0 & -r \sin \phi
\end{array}\right] } \\
& \times\left[\begin{array}{ccc}
\cos \theta \sin \phi & \sin \theta \sin \phi & \cos \phi \\
-r^{-1} \sin \theta / \sin \phi & r^{-1} \cos \theta / \sin \phi & 0 \\
r^{-1} \cos \theta \cos \phi & r^{-1} \sin \theta \cos \phi & -r^{-1} \sin \phi
\end{array}\right] \\
= & {\left[\begin{array}{ccc}
\cos g & -\sin g & 0 \\
\sin g & \cos g & 0 \\
0 & 0 & 1
\end{array}\right]+r g^{\prime}\left[\begin{array}{c}
-\sin \omega \\
\cos \omega \\
0
\end{array}\right] \otimes\left[\begin{array}{c}
\cos \theta \sin ^{2} \phi \\
\sin \theta \sin ^{2} \phi \\
\sin \phi \cos \phi
\end{array}\right] . }
\end{aligned}
$$

Hence as a result we have that

$$
|\nabla u|^{2}=3+r^{2} g^{\prime 2} \sin ^{2} \phi .
$$


Therefore in view of $|u|=r$ we can write

$$
\begin{aligned}
\int_{\Omega}|u|^{2}+|\nabla u|^{2} & =\int_{0}^{\pi} \int_{0}^{2 \pi} \int_{a}^{b}\left[3+r^{2}\left(1+g^{\prime 2} \sin ^{2} \phi\right)\right] r^{2} \sin \phi d r d \theta d \phi \\
& =4 \pi \int_{a}^{b}\left[3+r^{2}\left(1+\frac{2}{3} g^{\prime 2}\right)\right] r^{2} d r
\end{aligned}
$$

and so referring to (a) the conclusion follows.

REMARK 7.3. Similar to the case in two dimensions, when speaking of a twist $u$, we agree to set $g(a)=0$ and $g(b)=2 \pi\left(n_{b}-n_{a}\right)=2 \pi k$.

REmaRK 7.4. When $n \geq 3, \mathcal{A}(\Omega)$ does not embed into $C(\bar{\Omega}, \bar{\Omega})$. However, any twist $u$ is continuous. In fact, as a result of Proposition 7.2, every bounded sequence of twists $\left(u_{j}\right)_{j \in \mathbb{N}} \subset \mathcal{A}(\Omega)$ admits a uniformly convergent subsequence on $\bar{\Omega}$.

The difference between the cases $n=2$ and $n \geq 3$ is twofold; firstly, as indicated in Remark 7.4 Sobolev maps $u \in \mathcal{A}$, in general, do not have continuous representatives and secondly, the space of continuous self-maps of annuli have gravely different structure of homotopy classes in passing from $n=2$ to $n \geq 3$. To examine this more closely let $\Omega=\left\{x \in \mathbb{R}^{3}: a<|x|<b\right\}$ and consider the space

$$
\mathfrak{A}:=\{\phi \in C(\bar{\Omega}, \bar{\Omega}): \phi(x)=x \text { for } x \in \partial \Omega\},
$$

equipped with the topology of uniform convergence. Then, it can be shown that the homotopy classes of $\mathfrak{A}$ can be enumerated by $\mathbb{Z}_{2}$, i.e. the cyclic group of order 2 (see Proposition 8.2). In particular a pair of twists $u_{1}, u_{2} \in \mathfrak{A}$ are homotopic if and only if for their twist functions $g_{1}, g_{2}$ the corresponding integers $k_{1}, k_{2}$ satisfy $k_{2}-k_{1} \equiv 0(\bmod 2)$.

The natural question emerging here is if like the case $n=2$ the EulerLagrange equations (7.1) admit solutions in the form of twists? Motivated by the approach in Section 5, we proceed by considering the energy of a twist given by

$$
\begin{aligned}
\frac{1}{2} \int_{\Omega}|\nabla u|^{2} & =\int_{0}^{\pi} \int_{0}^{2 \pi} \int_{a}^{b}\left[\frac{3}{2}+\frac{1}{2} r^{2} g^{\prime 2} \sin ^{2} \phi\right] r^{2} \sin \phi d r d \theta d \phi \\
& =4 \pi \int_{a}^{b}\left[\frac{3}{2}+\frac{1}{3} r^{2} g^{\prime 2}\right] r^{2} d r .
\end{aligned}
$$

Then, setting

$$
\mathbb{E}[g]:=\int_{a}^{b}\left[\frac{3}{2}+\frac{1}{3} r^{2} g^{\prime 2}\right] r^{2} d r
$$


over the same space (5.1), it is a trivial matter to verify that the Euler-Lagrange equation corresponding to $\mathbb{E}$ takes the form

$$
\frac{d}{d r}\left[r^{4} g^{\prime}(r)\right]=0
$$

which together with the boundary conditions $g(a)=0$ and $g(b)=2 k \pi$ admits the unique (minimizing) solution $g$ given by

$$
g(r)=2 k \pi \frac{a^{3} b^{3}}{b^{3}-a^{3}}\left[\frac{1}{a^{3}}-\frac{1}{r^{3}}\right] .
$$

However, surprisingly, we have the following negative result on the existence of twist solutions to the Euler-Lagrange equations (7.1).

Proposition 7.5. The Euler-Lagrange equations (7.1) do not admit twist solutions except for $u=x$.

Proof.

$$
\Delta u=\left[\begin{array}{c}
\Delta u_{1} \\
\Delta u_{2} \\
\Delta u_{3}
\end{array}\right]=\left[\begin{array}{c}
-4 g^{\prime} \sin \omega \sin \phi-r g^{\prime \prime} \sin \omega \sin \phi-r g^{2} \cos \omega \sin \phi \\
4 g^{\prime} \cos \omega \sin \phi+r g^{\prime \prime} \cos \omega \sin \phi-r g^{\prime 2} \sin \omega \sin \phi \\
0
\end{array}\right] .
$$

Hence, substitution in (7.1) gives

$$
\left[\begin{array}{l}
\mathfrak{p}_{, 1} \\
\mathfrak{p}_{, 2} \\
\mathfrak{p}_{, 3}
\end{array}\right]=\left[\begin{array}{c}
-\left(4 g^{\prime}+r g^{\prime \prime}\right) \sin \phi \sin \theta+\left\{\left(4 r g^{2}+r^{2} g^{\prime} g^{\prime \prime}\right) \sin ^{3} \phi-r g^{2} \sin \phi\right\} \cos \theta \\
\left(4 g^{\prime}+r g^{\prime \prime}\right) \sin \phi \cos \theta+\left\{\left(4 r g^{\prime 2}+r^{2} g^{\prime} g^{\prime \prime}\right) \sin ^{3} \phi-r g^{2} \sin \phi\right\} \sin \theta \\
\left(4 r g^{\prime 2}+r^{2} g^{\prime} g^{\prime \prime}\right) \sin ^{2} \phi \cos \phi
\end{array}\right] .
$$

or alternatively

$$
\left[\begin{array}{c}
\mathfrak{p}_{, r} \\
\mathfrak{p}_{, \theta} \\
\mathfrak{p}_{, \phi}
\end{array}\right]=\left[\begin{array}{c}
r g^{\prime}\left(3 g^{\prime}+r g^{\prime \prime}\right) \sin ^{2} \phi \\
r\left(4 g^{\prime}+r g^{\prime \prime}\right) \sin ^{2} \phi \\
-r^{2} g^{\prime 2} \sin \phi \cos \phi
\end{array}\right]
$$

As a necessary condition for the solvability of (7.2) is for the expression on the right to be curl-free we arrive at the set of equations

$$
\begin{aligned}
\frac{\partial}{\partial r}\left[r\left(4 g^{\prime}+r g^{\prime \prime}\right) \sin ^{2} \phi\right]-\frac{\partial}{\partial \theta}\left[r g^{\prime}\left(3 g^{\prime}+r g^{\prime \prime}\right) \sin ^{2} \phi\right] & =0, \\
\frac{\partial}{\partial \theta}\left[r^{2} g^{2} \sin \phi \cos \phi\right]+\frac{\partial}{\partial \phi}\left[r\left(4 g^{\prime}+r g^{\prime \prime}\right) \sin ^{2} \phi\right] & =0, \\
\frac{\partial}{\partial \phi}\left[r g^{\prime}\left(3 g^{\prime}+r g^{\prime \prime}\right) \sin ^{2} \phi\right]+\frac{\partial}{\partial r}\left[r^{2} g^{\prime 2} \sin \phi \cos \phi\right] & =0,
\end{aligned}
$$

or equivalently that

$$
\left\{\begin{array}{l}
r g^{\prime \prime}+4 g^{\prime}=0 \\
2 r g^{\prime} g^{\prime \prime}+4 g^{2}=0
\end{array}\right.
$$

However, this gives $g^{\prime \prime}=0, g^{\prime}=0$, which along with the boundary conditions $g(a)=0$ and $g(b)=2 k \pi$ admits the solution $g=0$ and indeed only so when we have that $k=0$. 
REMARK 7.6. The non-existence results, here, fail if the Dirichlet energy (1.1) is replaced by the so-called $p$-energy

$$
u \mapsto \frac{1}{p} \int_{\Omega}|\nabla u(x)|^{p} d x,
$$

for some $p \geq n$ and over the space

$$
\left\{u \in W^{1, p}\left(\Omega, \mathbb{R}^{n}\right):\left.u\right|_{\partial \Omega}=\varphi, \operatorname{det} \nabla u=1 \mathcal{L}^{n}-\text { a.e. in } \Omega\right\}
$$

Indeed, the energy functional (7.3) has two local minimizers (with respect to the $L^{1}$-metric) one associated to each of the components of $\mathfrak{A}$. For further results of this nature, we refer the reader to [13] and [14].

\section{Appendix}

8.1. Continuous self-maps of annuli. Let $\Omega=\left\{x \in \mathbb{R}^{n}: a<|x|<b\right\}$ with $0<a<b<\infty$ and consider the space

$$
\mathfrak{A}:=\{\phi \in C(\bar{\Omega}, \bar{\Omega}): \phi(x)=x \text { for } x \in \partial \Omega\},
$$

equipped with the topology of uniform convergence.

Definition 8.1 (Homotopy). A pair of maps $\phi_{0}, \phi_{1} \in \mathfrak{A}$ are referred to as homotopic if and only if there exists a continuous map $h:[0,1] \times \bar{\Omega} \rightarrow \bar{\Omega}$ such that

(a) $h(0, x)=\phi_{0}(x)$ for all $x \in \bar{\Omega}$,

(b) $h(1, x)=\phi_{1}(x)$ for all $x \in \bar{\Omega}$,

(c) $h(t, x)=x$ for all $t \in[0,1]$ and $x \in \partial \Omega$.

The collection of all maps homotopic to $\phi \in \mathfrak{A}$ is referred to as the homotopy class of $\phi$ and denoted by $[\phi]$.

Proposition 8.2. he set $\{[\phi]: \phi \in \mathfrak{A}\}$ can be enumerated by

(a) $\mathbb{Z} \cong \pi_{1}\left[C_{\varphi}\left(\mathbb{S}^{1}, \mathbb{S}^{1}\right)\right]$ when $n=2$, and

(b) $\mathbb{Z}_{2} \cong \pi_{1}\left[C_{\varphi}\left(\mathbb{S}^{n-1}, \mathbb{S}^{n-1}\right)\right]$ when $n \geq 3$. (13)

Proof. See [13, pp. 391-393].

Proposition 8.3. Let $\phi_{0}, \phi_{1} \in \mathfrak{A}$ and $\left|\phi_{0}-\phi_{1}\right|<a$, uniformly on $\bar{\Omega}$. Then $\left[\phi_{0}\right]=\left[\phi_{1}\right]$.

$\left.{ }^{13}\right)$ Here $\varphi$ denotes the identity map of the $m$-sphere and $C_{\varphi}\left(\mathbb{S}^{m}, \mathbb{S}^{m}\right)$ is the pathconnected component of $C\left(\mathbb{S}^{m}, \mathbb{S}^{m}\right)$ containing $\varphi$. 
Proof. Consider the (continuous) projection map $\pi_{\bar{\Omega}}: \mathbb{R}^{n} \backslash\{0\} \rightarrow \bar{\Omega}$ defined via $\pi_{\bar{\Omega}}(x)=\rho(|x|) x$, where

$$
\rho(s)= \begin{cases}a s^{-1} & \text { for } 0<s \leq a \\ 1 & \text { for } a \leq s \leq b \\ b s^{-1} & \text { for } b \leq s\end{cases}
$$

Then, the homotopy $h:[0,1] \times \bar{\Omega} \rightarrow \bar{\Omega}$ defined via

$$
h(t, x):=\pi_{\bar{\Omega}}\left[(1-t) \phi_{0}(x)+t \phi_{1}(x)\right]
$$

is well-defined and satisfies all the assumptions in Definition 8.1. As a result $\left[\phi_{0}\right]=\left[\phi_{1}\right]$ and so the conclusion follows.

Proposition 8.4. For each $\phi \in \mathfrak{A},[\phi] \subset \mathfrak{A}$ is both open and closed with respect to the topology of uniform convergence.

Proof. This is an immediate consequence of Proposition 8.3.

8.2. Degree of maps on two dimensional annuli. Consider now the case $n=2$. Referring to (a) in Proposition 8.2, here, we intend to give a characterisation of the integer through which the homotopy class $[\phi]$ can be described.

Indeed fix $\phi \in \mathfrak{A}$. Then using polar coordinates for $\theta \in[0,2 \pi]$ (fixed) the $\mathbb{S}^{1}$-valued curve

$$
\gamma^{\theta}(r)=\frac{\phi}{|\phi|}(r, \theta):[a, b] \rightarrow \mathbb{S}^{1},
$$

has a well-defined index (or winding number about the origin). Furthermore, in view of continuity of $\phi$, this is independent of the particular choice of $\theta \in[0,2 \pi]$. The latter correspondence will be denoted by $\left({ }^{14}\right)$

$$
\phi \mapsto \operatorname{deg}\left(\frac{\phi}{|\phi|}\right) .
$$

Note that for a differentiable curve (taking advantage of $\mathbb{S}^{1} \subset \mathbb{C}$ ) we specifically have the formula

$$
\operatorname{deg}\left(\frac{\phi}{|\phi|}\right)=\frac{1}{2 \pi i} \int_{\gamma} \frac{d z}{z} .
$$

A straight-forward continuity argument combined with the fact that $\operatorname{deg}(\cdot)$ is integer-valued gives

$$
\left[\phi_{0}\right]=\left[\phi_{1}\right] \Rightarrow \operatorname{deg}\left(\frac{\phi_{0}}{\left|\phi_{0}\right|}\right)=\operatorname{deg}\left(\frac{\phi_{1}}{\left|\phi_{1}\right|}\right)
$$

In particular we have the following convergence result.

(14) This integer also agrees with the Brouwer degree of the map resulting from identifying $\mathbb{S}^{1} \cong[a, b] /\{a, b\}$, justified as a result of $\gamma_{\theta}(a)=\gamma_{\theta}(b)$. 
Proposition 8.5. Let $\left(\phi_{j}\right)_{j \in \mathbb{N}} \subset \mathfrak{A}$ and $\phi_{j} \rightarrow \phi$ uniformly on $\bar{\Omega}$. Then $\phi \in \mathfrak{A}$ and

$$
\operatorname{deg}\left(\frac{\phi_{j}}{\left|\phi_{j}\right|}\right) \rightarrow \operatorname{deg}\left(\frac{\phi}{|\phi|}\right)
$$

Proof. The first assertion from the closedness of $\bar{\Omega}$ and the convergence follows from Proposition 8.3 together with (8.1).

That the converse to (8.1) is also true is the content of the following proposition.

Proposition 8.6. Let $\phi_{0}, \phi_{1} \in \mathfrak{A}$ and $\operatorname{deg}\left(\phi_{0} /\left|\phi_{0}\right|\right)=\operatorname{deg}\left(\phi_{1} / \mid \phi_{1}\right)$. Then $\left[\phi_{0}\right]=\left[\phi_{1}\right]$.

Proof. Fix $\phi_{0}, \phi_{1} \in \mathfrak{A}$. The conclusion will follow once we construct a homotopy between $\phi_{0}$ and $\phi_{1}$. Since, by assumption, the restrictions of $\phi_{0}$ and $\phi_{1}$ to any radial ray have the same index (viewed as maps from $\mathbb{S}^{1}$ to $\mathbb{S}^{1}$ have the same degree) it follows from Brouwer-Hopf theorem that the latter are homotopic. More specifically, for each fixed $\theta \in[0,2 \pi]$, there exists $h^{\theta}:[0,1] \times$ $[a, b] \rightarrow \mathbb{S}^{1}$ such that,

(a) $h^{\theta}(0, r)=\gamma_{0}^{\theta}(r)$ for all $r \in[a, b]$,

(b) $h^{\theta}(1, r)=\gamma_{1}^{\theta}(r)$ for all $r \in[a, b]$,

(c) $h^{\theta}(t, r)=1$ for all $t \in[0,1]$ and $r \in\{a, b\}$.

Now to proceed further for any fixed pair of $\theta_{0}, \theta_{1} \in[0,2 \pi]$ set

$$
\Omega\left(\theta_{0}, \theta_{1}\right):=\left\{x=(r, \theta): a<r<b \text { and } \theta_{0}<\theta<\theta_{1}\right\} .
$$

Then we consider the continuous map

$$
H: \partial\left[[0,1] \times \bar{\Omega}\left(\theta_{0}, \theta_{1}\right)\right] \rightarrow \mathbb{S}^{1},
$$

defined for $t \in\{0,1\}$ and $x \in \bar{\Omega}\left(\theta_{0}, \theta_{1}\right)$ via

$$
H\left(\theta_{0}, \theta_{1}, t, x\right)= \begin{cases}\phi_{0}(x) & \text { for } t=0, \\ \phi_{1}(x) & \text { for } t=1,\end{cases}
$$

and for $t \in[0,1]$ and $x \in \partial \Omega\left(\theta_{0}, \theta_{1}\right)$ via

$$
H\left(\theta_{0}, \theta_{1}, t, x\right)= \begin{cases}a^{-1} x & \text { for } r=a, \theta \in\left[\theta_{0}, \theta_{1}\right], \\ h^{\theta_{0}}(t, x) & \text { for } \theta=\theta_{0}, r \in[a, b], \\ h^{\theta_{1}}(t, x) & \text { for } \theta=\theta_{1}, r \in[a, b], \\ b^{-1} x & \text { for } r=b, \theta \in\left[\theta_{0}, \theta_{1}\right] .\end{cases}
$$

Since $\pi_{2}\left(\mathbb{S}^{1}\right) \cong 0$, the obstruction to extending the latter map (from the boundary to the interior of $\left.[0,1] \times \bar{\Omega}\left(\theta_{0}, \theta_{1}\right)\right)$ vanishes and so $H$ can be extended (again, denoted $H)$ to $H:[0,1] \times \bar{\Omega}\left(\theta_{0}, \theta_{1}\right) \rightarrow \mathbb{S}^{1}$. 
In order to complete the proof it suffices to take $\rho:[0,1] \times \bar{\Omega}\left(\theta_{0}, \theta_{1}\right) \rightarrow[a, b]$ defined via

$$
\rho(t, x)=(1-t)\left|\phi_{0}(x)\right|+t\left|\phi_{1}(x)\right|
$$

and finally to set

$$
h(t, x)=\rho(t, x) \times \begin{cases}H(0, \pi, t, x) & \text { for } x \in \bar{\Omega}(0, \pi), \\ H(\pi, 2 \pi, t, x) & \text { for } x \in \bar{\Omega}(\pi, 2 \pi) .\end{cases}
$$

Then $h$ is the required homotopy.

As a result of $(8.1) \operatorname{deg}(\cdot)$ induces a map (again, denoted deg) on the set $\{[\phi]: \phi \in \mathfrak{A}\}$. In conjunction with Proposition 8.9 this leads us to the following conclusion.

TheOrem 8.7. Let $n=2$. Then, the $\operatorname{map} \operatorname{deg}(\cdot):\{[\phi]: \phi \in \mathfrak{A}\} \rightarrow \mathbb{Z}$ is a bijection.

8.3. $\mathrm{SO}(3)$ and maps on three dimensional annuli. Consider now the case $n=3$. Referring to (b) in Proposition 8.2, here, with the aid of the fundamental group of $\mathrm{SO}(3)$ we intend to give a characterisation of the homotopy classes $\{[\phi]: \phi \in \mathfrak{A}\}$.

To this end fix $\phi \in \mathfrak{A}$. Then using the identification $\bar{\Omega} \cong[a, b] \times \mathbb{S}^{2}$ it is plain that the map

$$
\omega[r](\cdot)=\frac{\phi}{|\phi|}(r, \cdot):[a, b] \rightarrow C_{\varphi}\left(\mathbb{S}^{2}, \mathbb{S}^{2}\right),
$$

uniquely defines an element of the group $\pi_{1}\left[C_{\varphi}\left(\mathbb{S}^{2}, \mathbb{S}^{2}\right)\right]$. The latter correspondence will be denoted by

$$
\phi \mapsto \operatorname{deg}_{2}\left(\frac{\phi}{|\phi|}\right) \in \mathbb{Z}_{2}
$$

Consider now the action of $\mathrm{SO}(3)$ on $\mathbb{S}^{2}$ [viewed as the group of orientation preserving isometries of $\mathbb{S}^{2}$ onto itself], i.e. $\mathbf{E}: \xi \in \mathrm{SO}(3) \mapsto \omega \in C\left(\mathbb{S}^{2}, \mathbb{S}^{2}\right)$ where $\mathbf{E}[\omega](x)=\xi x$.

Proposition 8.8. With the above notation, the induced homomorphism

$$
\mathbf{E}^{*}: \pi_{1}[\mathrm{SO}(3)] \rightarrow \pi_{1}\left[C_{\varphi}\left(\mathbb{S}^{2}, \mathbb{S}^{2}\right)\right]
$$

is an isomorphism.

Proof. It is not hard to see that evaluation at base point defines a Hurewicz fibration $q: \mathrm{SO}(3) \rightarrow \mathbb{S}^{2}$ with the corresponding fibre $q^{-1}(1)=\mathrm{SO}(2)$. Thus, we 
are lead to the following commutative diagram between fibrations $\left({ }^{15}\right)$ :

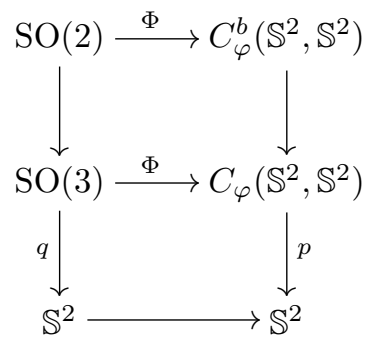

It is well-known that $\pi_{1}(\mathrm{SO}(2)) \cong \mathbb{Z}$ and $\pi_{1}(\mathrm{SO}(3)) \cong \mathbb{Z}_{2}$. In a similar way,

$$
\pi_{1}\left[\mathbf{C}_{\varphi}^{b}\left(\mathbb{S}^{2}, \mathbb{S}^{2}\right)\right] \cong \pi_{1}\left[\mathbb{S}^{2}\right] \oplus \pi_{3}\left(\mathbb{S}^{2}\right) \cong \mathbb{Z}
$$

Thus, an easy inspection of the exact homotopy sequences corresponding to the above diagram together with the fact that the homomorphism induced by the inclusion of $\mathrm{SO}(2)$ into $\mathrm{SO}(3)$ maps the generator of $\pi_{1}[\mathrm{SO}(2)]$ to the non-zero element in $\pi_{1}[\mathrm{SO}(3)]$ implies the assertion. $\left({ }^{16}\right)$

Proposition 8.9. Let $\phi_{0}, \phi_{1} \in \mathfrak{A}$ and $\operatorname{deg}_{2}\left(\phi_{0} /\left|\phi_{0}\right|\right)=\operatorname{deg}_{2}\left(\phi_{1} / \mid \phi_{1}\right)$. Then $\left[\phi_{0}\right]=\left[\phi_{1}\right]$.

Proof. Fix $\phi_{0}, \phi_{1} \in \mathfrak{A}$ and let $\gamma_{0}=\phi_{0} /\left|\phi_{0}\right|, \gamma_{1}=\phi_{1} /\left|\phi_{1}\right|$. It then follows from the hypotheses that $\gamma_{0}, \gamma_{1}$ represent the same elements of $\pi_{1}\left[C_{\varphi}\left(\mathbb{S}^{2}, \mathbb{S}^{2}\right)\right]$ and so there exists a continuous map $H:[0,1] \times \bar{\Omega} \rightarrow \mathbb{S}^{2}$ so that

(a) $H(0, x)=\gamma_{0}(x)$ for all $x \in \bar{\Omega}$,

(b) $H(1, x)=\gamma_{1}(x)$ for all $x \in \bar{\Omega}$,

(c) $H(t, x)=x /|x|$ for all $t \in[0,1]$ and $x \in \partial \Omega$.

With the introduction of the map $H$ above consider now the map $\rho:[0,1] \times$ $\bar{\Omega} \rightarrow[a, b]$ defined via

$$
\rho(t, x)=(1-t)\left|\phi_{0}(x)\right|+t\left|\phi_{1}(x)\right| .
$$

Then, the required homotopy between $\phi_{0}, \phi_{1}$ is given by,

$$
h(t, x)=\rho(t, x) H(t, x) .
$$

This complete the proof.

$\left({ }^{15}\right)$ Here, $C_{\varphi}^{b}\left(\mathbb{S}^{2}, \mathbb{S}^{2}\right)$ denotes the components containing $\varphi$ in the space of base-preserving maps.

(16) Indeed, it is well-known that the cyclic group $\pi_{1}[\mathrm{SO}(3)] \cong \mathbb{Z}_{2}$ is generated by the rotations about a fixed axis by $2 \pi$, e.g. the homotopy class of the closed curve

$$
\theta \in[0,2 \pi] \mapsto\left[\begin{array}{ccc}
\cos \theta & \sin \theta & 0 \\
-\sin \theta & \cos \theta & 0 \\
0 & 0 & 1
\end{array}\right] \in \mathrm{SO}(3)
$$

denoted $[\mathbf{1}] \in \mathbb{Z}_{2}$. (See e.g. [3, pp. 164-165]). 
It remains therefore to give a complete enumeration of the homotopy classes $\{[\phi]: \phi \in \mathfrak{A}\}$. As we evidently have the implication

$$
\left[\phi_{0}\right]=\left[\phi_{1}\right] \Rightarrow \operatorname{deg}_{2}\left(\frac{\phi_{0}}{\left|\phi_{0}\right|}\right)=\operatorname{deg}_{2}\left(\frac{\phi_{1}}{\left|\phi_{1}\right|}\right),
$$

we are lead to the following conclusion.

TheOREM 8.10. Let $n=3$. Then, the $\operatorname{map} \operatorname{deg}_{2}(\cdot):\{[\phi]: \phi \in \mathfrak{A}\} \rightarrow \mathbb{Z}_{2}$ is a bijection.

Let $u$ be a three dimensional twist with a corresponding twist function $g$. Then, it is a trivial matter to verify that

$$
\operatorname{deg}_{2}\left(\frac{u}{|u|}\right)= \begin{cases}{[\mathbf{0}]} & \text { for } k \text { even } \\ {[\mathbf{1}]} & \text { for } k \text { odd }\end{cases}
$$

Therefore, the two homotopy classes of $\mathfrak{A}$ contain all twists, with one containing those corresponding to $k$ being even and the other with $k$ being odd. In particular a pair of twists $u_{1}, u_{2}$ are homotopic if and only if for the corresponding twist functions $g_{1}, g_{2}$ we have that $k_{2}-k_{1} \equiv 0 \bmod 2$.

\section{REFERENCES}

[1] J. M. BALL, Convexity conditions and existence theorems in nonlinear elasticity, Arch. Rational Mech. Anal. 63 (1977), 337-403.

[2] P. Bauman, N. C. Owen and D. Phillips, Maximum principles and a priori estimates for an incompressible material in nonlinear elasticity, Comm. Partial Differential Equations 17 (1992), 1185-1212.

[3] G. Bredon, Topology and Geometry, Grad. Texts in Math., vol. 139, Springer, 1993.

[4] R. Coifman, P. L. Lions, Y. Meyer and S. Semmes, Compensated compactness and Hardy spaces, J. Math. Pures Appl. 72 (1993), 247-286.

[5] M. Denn, Die Gruppe der Abbildungsklassen, Acta Math. 69 (1938), 135-206.

[6] L. C. Evans And R. F. GaRiepy, On the partial regularity of energy-minimizing, area preserving maps, Calc. Var. 63 (1999), 357-372.

[7] H. LE DRET, Constitutive laws and existence questions in incompressible nonlinear elasticity, J. Elasticity 15 (1985), 369-387.

[8] C. B. Morrey, Multiple Integrals in the Calculus of Variations, Grad. Texts in Math., vol. 130 Springer, 1966.

[9] S. Müller, Q. Tang and B. S. Yan, On a new class of elastic deformations not allowing for cavitation, Ann. Inst. H. Poincaré Anal. Non Linéaire 11 (1994), 217-243.

[10] K. Post and J. Sivaloganathan, On homotopy conditions and the existence of multiple equilibria in finite elasticity, Proc. Roy. Soc. Edinburgh Sect. A 127 (1997), 595-614.

[11] W. Rudin, Real and Complex Analysis, 3rd Edition,, McGraw Hill, 1987.

[12] A. TAHERI, On Artin's braid group and polyconvexity in the calculus of variations, J. London Math. Soc. 67 (2003), 752-768.

[13] Local minimizers and quasiconvexity - the impact of topology, Arch. Rational Mech. Anal. 176 (2005), 363-414. 
[14] _ Stationary twists and energy minimizers on a space of measure preserving maps, submitted for publication (2008).

[15] S. K. Vodopyanov and V. M. Gol'Dshtein, Quasiconformal mappings and spaces of functions with generalized first derivatives, Siberian Math. J. 17 (1977), 515-531.

[16] K. ZHANG, On the coercivity of elliptic systems in two dimensions, Bull. Australian Math. Soc. 54 (1996), 423-430.

Manuscript received May 15, 2008

Ali TAheri

Department of Mathematics

University of Sussex

Falmer BN1 9FR, UNITED KINGDOM

E-mail address: a.taheri@sussex.ac.uk 